Dieses Dokument ist eine Zweitveröffentlichung (Verlagsversion) / This is a self-archiving document (published version):

Tian-Yi Li, You-Xuan Zheng, Yong-Hui Zhou

Iridium(III) phosphorescent complexes with dual stereogenic centers: single crystal, electronic circular dichroism evidence and circularly polarized luminescence properties

Erstveröffentlichung in / First published in:

Dalton Transactions. 2016, 45(48), S. 19234-19237 [Zugriff am: 01.11.2019]. Royal Society of Chemistry. ISSN 1477-9234.

DOI: https://doi.org/10.1039/c6dt04030f

Diese Version ist verfügbar / This version is available on:

https://nbn-resolving.org/urn:nbn:de:bsz:14-qucosa2-361230

„Dieser Beitrag ist mit Zustimmung des Rechteinhabers aufgrund einer (DFGgeförderten) Allianz- bzw. Nationallizenz frei zugänglich.“

This publication is openly accessible with the permission of the copyright owner. The permission is granted within a nationwide license, supported by the German Research Foundation (abbr. in German DFG).

www.nationallizenzen.de/ 
CrossMark

\&lick for updates

Cite this: Dalton Trans., 2016, 45 19234

Received 20th October 2016, Accepted 11th November 2016

DOI: $10.1039 / c 6 d t 04030 f$

www.rsc.org/dalton

\title{
Iridium(III) phosphorescent complexes with dual stereogenic centers: single crystal, electronic circular dichroism evidence and circularly polarized luminescence properties $\uparrow$
}

\author{
Tian-Yi Li, ${ }^{\text {a,b }}$ You-Xuan Zheng*a and Yong-Hui Zhou*c
}

Iridium complexes with a chiral metal center and chiral carbons, $\Lambda / \Delta$-(dfppy $)_{2} \operatorname{Ir}(\text { chty- } R \text { ) and } \Lambda / \Delta \text {-(dfppy) })_{2} \operatorname{Ir}($ chty-S), were synthesized and characterized. These isomers have the same steadystate photophysical properties, and obvious offsets in ECD spectra highlight both the chiral sources. Each enantiomeric couple shows mirror-image CPL bands with a dissymmetry factor in the order of $10^{-3}$.

As promising phosphorescent emitters, Ir(III) octahedral complexes with 2-phenylpyridine analogue ligands and various ancillary ligands have been investigated extensively. ${ }^{1}$ With tunable photophysical properties, many of these complexes have been applied in organic light-emitting diodes (OLEDs), ${ }^{2}$ chemical sensing ${ }^{3}$ and biological probing. ${ }^{4}$ Due to the nature of six-coordinated complexes, these $\operatorname{Ir}(\mathrm{III})$ complexes have isomers with two configurations, which are marked as $\Lambda$ and $\Delta .^{5}$ According to the well-established synthetic route via a chloro-bridge dimer, a mixture of an equal molar ratio of $\Lambda$ and $\Delta$ isomers is obtained. However, these two isomers present exactly the same steady-state photophysical properties, and the racemoids do not influence their applications in the fields mentioned above.

Recently, a growing number of publications have focused on the separation, characterization and application of $\operatorname{Ir}(\mathrm{III})$ phosphor isomers. ${ }^{6}$ In a previous study, we demonstrated that almost all conventional $\operatorname{Ir}($ III) phosphors with the structure of

\footnotetext{
${ }^{a}$ State Key Laboratory of Coordination Chemistry, Collaborative Innovation Center of Advanced Microstructures, School of Chemistry and Chemical Engineering, Nanjing University, Nanjing 210093, P. R. China.E-mail: yxzheng@nju.edu.cn ${ }^{b}$ Dresden Integrated Center for Applied Physics and Photonic Materials, Technische Universität Dresden, George-Bähr-Str.1, 01069 Dresden, Germany cJiangsu Collaborative Innovation Center of Atmospheric Environment and Equipment Technology, Jiangsu Key Laboratory of Atmospheric Environment Monitoring and Pollution Control, College of Environmental Science and Engineering, Nanjing University of Information Science \& Technology, Nanjing 210044, P. R. China.E-mail:yhzhou2007@aliyun.com $\dagger$ Electronic supplementary information (ESI) available: Synthetic details, single crystal data, theoretical calculations and more ECD and CPL information. CCDC 1033811-1033814. For ESI and crystallographic data in CIF or other electronic format see DOI: $10.1039 /$ c6dt04030f
}

fac/mer- $\operatorname{Ir}\left(\mathrm{L}_{\mathrm{m}}\right)_{3}$ and $\left(\mathrm{L}_{\mathrm{m}}\right)_{2} \operatorname{Ir}\left(\mathrm{L}_{\mathrm{a}}\right)$ (where $\mathrm{L}_{\mathrm{m}}$ is the main ligand and $\mathrm{L}_{\mathrm{a}}$ is the ancillary ligand) can be separated into configurational isomers. ${ }^{7}$ Obvious circularly polarized luminescence (CPL) signals were observed for each pair of enantiomers. It is well known that CPL has potential application in various fields, and control of the polarisation of light is essential for optical data processing and display devices such as optical data storage, ${ }^{8}$ highly efficient three-dimensional displays and liquid crystal display backlights, ${ }^{9}$ effective spin sources in optical spintronics, ${ }^{10}$ optical quantum information processing and communication, ${ }^{11}$ and information carriers in quantum computation $^{12}$ as well as the induction of exotic quantum phenomena such as the Floquet topological state. ${ }^{13}$ However, research studies for the complexes with dual stereogenic centers are still rare. Recently, Abbate et al. reported a series of Ir(III) ionic complexes with a chiral metal center and chiral ancillary ligands, which show obvious vibrational circular dichroism (VCD). ${ }^{14}$

In this contribution, we synthesized two Schiff base ancillary ligands, chty- $R$ and chty- $S$ containing a chiral carbon atom (Scheme $\mathrm{S} 1 \dagger$ and Scheme 1). Then, Ir(III) complexes (dfppy) ${ }_{2} \mathrm{Ir}$ $(\text { chty- } R \text { ) and (dfppy) })_{2} \operatorname{Ir}($ chty- $S$ ) with 2-(2,4-difluorophenyl)pyridine (dfppy) as the main ligand were synthesized. The chloro-

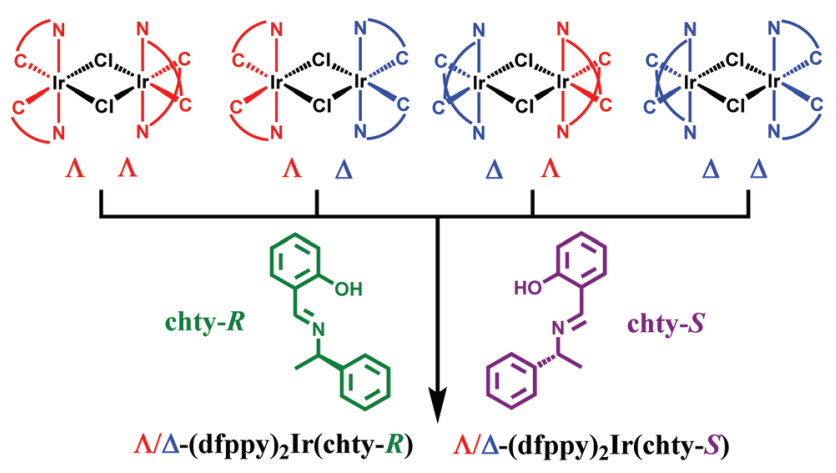

Scheme 1 Synthetic strategy for the isomers with chiral configurations and chiral carbon atoms. 
bridged dimer medium product was prepared by heating 2.2 equiv. of dfppy and 1 equiv. of iridium chloride in 2-ethoxyethanol at $120{ }^{\circ} \mathrm{C}$. The Schiff base ancillary ligands were synthesized according to the reported method starting from optically pure $R$ or $S$ isomers. ${ }^{15}$ For the final splitting reaction, 2.2 equiv. of Schiff base ancillary ligands, 1 equiv. of the dimer complex and 10 equiv. of potassium carbonate were heated at $120{ }^{\circ} \mathrm{C}$ in 2-ethoxyethanol overnight. ${ }^{16}$ The raw products were purified by chromatography and recrystallization to afford the final racemic complexes (dfppy $)_{2} \operatorname{Ir}(\mathrm{chty}-R)$ and (dfppy) $)_{2} \operatorname{Ir}($ chty- $S$ ) as yellow block crystals. Chiral synthetic HPLC was used to separate four isomers, termed $\Lambda / \Delta$ (dfppy) $)_{2} \operatorname{Ir}(\text { chty- } R \text { ) and } \Lambda / \Delta \text {-(dfppy })_{2} \operatorname{Ir}($ chty- $S$ ) hereafter, with ee values higher than $95 \%$ (Fig. S1 and $\mathrm{S} 2 \dagger$ ).

According to the refinement results of X-ray single crystal diffraction measurements, all the samples have a Flack index near zero which indicates that the absolute structures of the chiral sources are reliable. As illustrated in Fig. 1 and Table S2, $\uparrow$ the single crystal structures of four isomers leave no doubt with respect to the chirality assignment. As conventional Ir(III) phosphorescent complexes, all these four isomers adopt a twisted octahedral structure. The iridium center coordinates with two dfppy ligands via two trans nitrogen atoms and two cis carbon atoms and with the chiral Schiff base ancillary ligand chty- $R / S$ via an oxygen atom and a nitrogen atom. The Ir-C, Ir-N bonds between iridium and dfppy are around $2.0 \AA$ and $\mathrm{Ir}-\mathrm{O}$, Ir-N bonds with ancillary ligands are a bit longer around $2.1 \AA$ (Table S3†). The chirality on the carbon atom in the ancillary ligands is easy to distinguish by single crystal structures, and it is noteworthy that $\left[\Lambda-(\mathrm{dfppy})_{2} \operatorname{Ir}(\right.$ chty- $R)$, $\Delta$-(dfppy $)_{2} \operatorname{Ir}($ chty- $\left.S)\right]$ and $[\Delta \text {-(dfppy })_{2} \operatorname{Ir}($ chty- $R), \quad \Lambda$-(dfppy $)_{2} \operatorname{Ir}$ $($ chty-S)] are two couples of antimeres with perfect mirror symmetry structures. Due to a favourable steric position, the free phenyl rings connecting to the chiral carbon atom in $\Lambda$-(dfppy $)_{2} \operatorname{Ir}($ chty- $R)$ and $\Delta$-(dfppy $)_{2} \operatorname{Ir}($ chty- $S)$ present an obvious $\pi-\pi$ stacking effect with a pyridine ring in the dfppy ligand (Fig. S3†). The distance between two centers is around $3.9 \AA$. However, such stacking does not exist in $\Delta$-(dfppy $)_{2} \operatorname{Ir}($ chty- $R$ ) and $\Lambda$-(dfppy $)_{2} \operatorname{Ir}($ chty- $S)$. Consequently, $\Lambda$-(dfppy $)_{2} \operatorname{Ir}($ chty- $R$ ) and $\Delta$-(dfppy $)_{2} \operatorname{Ir}($ chty- $S$ ) crystallized in an orthorhombic crystal system with the $P 2{ }_{1} 2_{1} 2_{1}$ space group with higher symmetry than the $P 2_{1}$ space group in a monoclinic system which is adopted by $\Delta$-(dfppy $)_{2} \operatorname{Ir}($ chty- $R)$ and $\Lambda$-(dfppy $)_{2} \operatorname{Ir}($ chty- $S)$.

The steady state photophysical properties were measured in diluted $\mathrm{CH}_{2} \mathrm{Cl}_{2}$ solutions $\left(1 \times 10^{-5} \mathrm{M}\right.$ for absorption and $5 \times 10^{-5} \mathrm{M}$ for emission, respectively, Fig. 2). All the isomers present almost the same spectral profile which corresponds to similar research conducted earlier. ${ }^{17}$ This demonstrates that neither the chiral configuration nor the chiral atoms in the ligands influences the steady state absorption and emission properties. As conventional Ir(III) complex phosphors, each of these four compounds has an intensive absorption band peaking at $250 \mathrm{~nm}$, which is assigned to spin-allowed $\pi-\pi$ transitions. Another weak absorption band is observed covering a

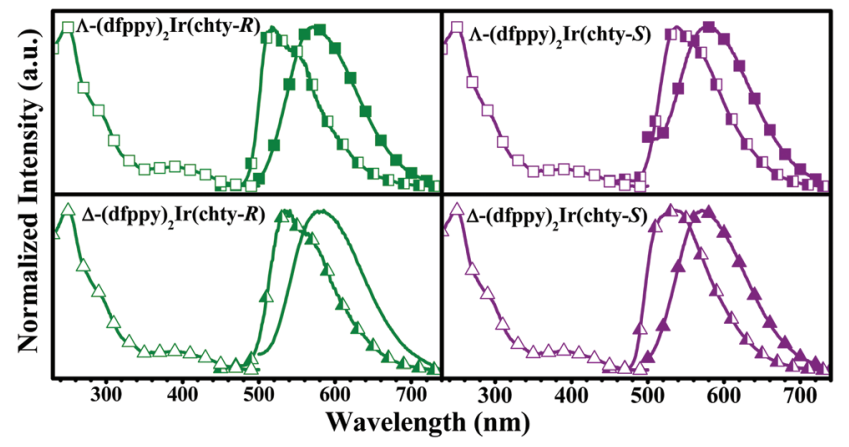

Fig. 2 The UV-vis absorption and photoluminescence spectra of all the isomers (absorption: empty, emission at $298 \mathrm{~K}$ : filled, emission at $77 \mathrm{~K}$ : half filled; lambda: square, delta: triangle).

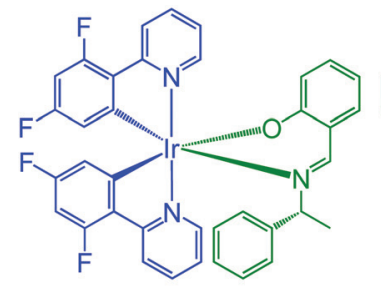

$\Lambda$-(dfppy $)_{2} \operatorname{Ir}($ chty- $R)$

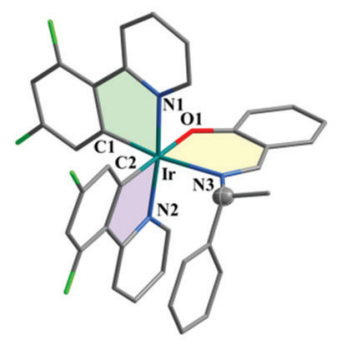

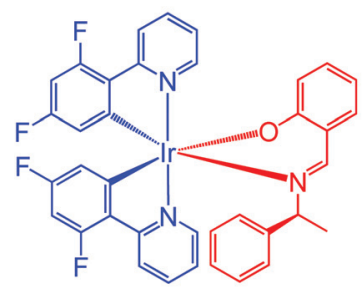

$\Lambda$-(dfppy $)_{2} \operatorname{Ir}($ chty-S)
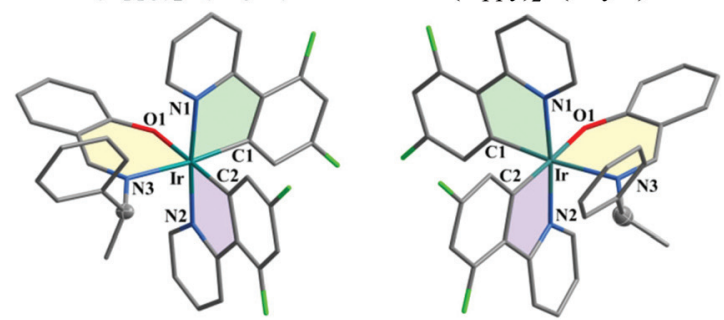

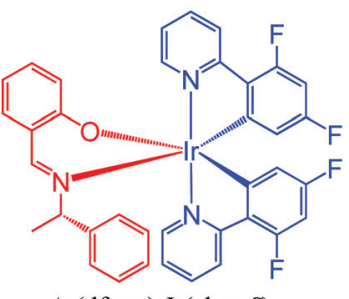

$\Delta$-(dfppy) $)_{2} \operatorname{Ir}($ chty- $S)$

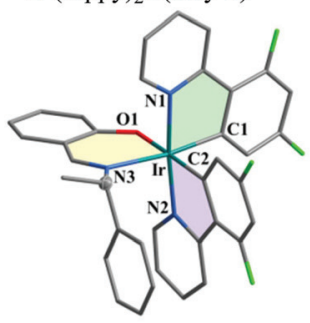

Fig. 1 The molecular structures (top) and single crystal diagrams (bottom) of $\Lambda / \Delta$-(dfppy) $\operatorname{Ir}($ chty- $R$ ) and $\Lambda / \Delta$-(dfppy) 2 Ir(chty-S); (carbon: gray; nitrogen: blue; fluorine: green, oxygen: red; iridium: teal). 
wide range from 350 to $500 \mathrm{~nm}$ and it is generated by MLCT transitions including ${ }^{1} \mathrm{MLCT}$ and ${ }^{3} \mathrm{MLCT}$ due to the strong spin-orbital coupling effect introduced by iridium atoms. The emission spectra of all the isomers at room temperature show a broad band covering a wide range from 500 to $700 \mathrm{~nm}$, peaking at $580 \mathrm{~nm}$, which makes them orange emitters. When measured at $77 \mathrm{~K}$, the emission bands have a hypsochromic shift and the main peaks are around $520 \mathrm{~nm}$. The broad emission spectra at low temperature indicate that the emitting state has more ${ }^{3}$ MLCT character than ${ }^{3}$ LLCT character. ${ }^{18}$

The ECD spectra of all the four isomers after chiral HPLC together with the non-separated racemic complexes after the chromatography were recorded in $\mathrm{CH}_{2} \mathrm{Cl}_{2}$ solutions (Fig. 3a and $\mathrm{S} 5 \dagger$ ). Since the electron circular dichroism (ECD) peaks of the two types of chiral sources were separated from each other, signals generated by both chiral configuration and the chiral carbon atoms can be distinguished clearly. For the racemic complexes, the configuration chirality is counteracted because of the $1: 1$ mixing ratio of $\Lambda$ and $\Delta$ isomers out of the synthesis from the chloro-bridged intermediate into racemic products. Thus, the ECD signals for these samples are exclusively from the chiral carbon atoms in the ancillary ligands. In the range from 350 to $480 \mathrm{~nm},(\Lambda / \Delta)-(\mathrm{dfppy})_{2} \operatorname{Ir}($ chty- $R)$ show a broad peak in the positive direction and $(\Lambda / \Delta)-(\mathrm{dfppy})_{2} \operatorname{Ir}($ chty- $S)$ show a perfect mirror-image peak in the negative direction. The peak values for the differences of the extinction coefficients between right and left circularly polarized light are 6.5 and $-4.6 \mathrm{M}^{-1} \mathrm{~cm}^{-1}$. In the range lower than $350 \mathrm{~nm}$, no response caused by chiral carbon atoms is observed. In the case of the isomers, the joint effects of both the chiral metal center and the chiral carbon atoms make the spectra more complicated. For $\Lambda$-(dfppy $)_{2} \operatorname{Ir}($ chty- $R)$ and $\Delta$-(dfppy $)_{2} \operatorname{Ir}($ chty- $R)$, in the range lower than $350 \mathrm{~nm}$, only signals from chiral configurations are observed, and the spectra in this part present a mirror-image relationship due to the chiral metal center. The peaks at $310 \mathrm{~nm}$ have $\Delta \varepsilon$ values around 20 and $-21 \mathrm{M}^{-1} \mathrm{~cm}^{-1}$ respectively. However, from $350 \mathrm{~nm}$ onward, influence from chiral carbon atoms starts to appear and the spectra are no longer symmetric, being disturbed by a positive shift from chiral carbon atoms. Due to the same concentration, the cross of the two curves is exactly on the line of the racemic mixture instead of on the horizontal axis. The same situation can be also observed in the $\Lambda$-(dfppy $)_{2} \operatorname{Ir}($ chty- $S), \Delta$-(dfppy $)_{2} \operatorname{Ir}($ chty- $S)$ and $(\Lambda / \Delta)-(\text { dfppy })_{2} \operatorname{Ir}($ chty- $S)$. The peak values for $\Lambda$-(dfppy $)_{2} \operatorname{Ir}$
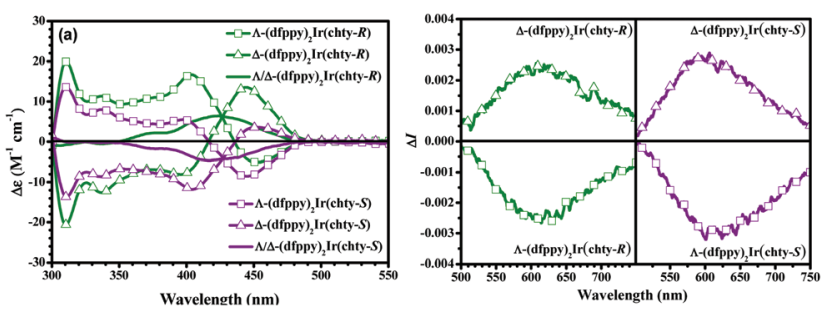

Fig. 3 The ECD spectra (a) and CPL spectra (b) of the four isomers in $\mathrm{CH}_{2} \mathrm{Cl}_{2}$ solutions.
$($ chty $-S)$ and $\Delta$-(dfppy $)_{2} \operatorname{Ir}($ chty- $S)$ at $310 \mathrm{~nm}$ are around \pm 14 $\mathrm{M}^{-1} \mathrm{~cm}^{-1}$. As a result, the spectra of $\Lambda$-(dfppy $)_{2} \operatorname{Ir}($ chty- $R)$ and $\Delta$-(dfppy $)_{2} \operatorname{Ir}($ chty- $S)$, and $\Delta$-(dfppy $)_{2} \operatorname{Ir}($ chty- $R)$ and $\Lambda$-(dfppy $)_{2} \operatorname{Ir}$ $($ chty- $S$ ) present crosses on the horizontal axis at 416 and $436 \mathrm{~nm}$ with $\Delta \varepsilon$ values of 6.3 and $4.3 \mathrm{M}^{-1} \mathrm{~cm}^{-1}$ respectively. It is noteworthy that the ECD signals from chiral carbon atoms in the Schiff base ancillary ligands are strong enough to generate positive or negative shifts on the ECD signals from chiral metal centers, making the ECD an available and efficacious method to characterize these isomers.

The CPL properties of the isomers were studied in $\mathrm{CH}_{2} \mathrm{Cl}_{2}$ solutions (Fig. 3b) as further chiroptical characterization. $\Lambda$-(dfppy $)_{2} \operatorname{Ir}($ chty- $R)$ and $\Delta$-(dfppy $)_{2} \operatorname{Ir}($ chty- $R$ ) have mirror-image profiles with a single band in the range from 500 to $750 \mathrm{~nm}$ peaking around $620 \mathrm{~nm}$. A similar relationship is observed for $\Lambda$-(dfppy $)_{2} \operatorname{Ir}($ chty- $S)$ and $\Delta$-(dfppy $)_{2} \operatorname{Ir}($ chty- $S)$. It is noteworthy that isomers with $\Delta$ configuration show positive CPL signals and the isomers with $\Lambda$ configuration give negative signals. Since no significant differences are observed in isomers with the same chiral configuration but different chiral carbon atoms, the CPL properties are mainly determined by the configurations, and the effects applied by the chiral carbon atoms in the ancillary ligands are negligible. The calculated dissymmetry factor $g_{\text {Lum }}$ is in the scope of $10^{-3}$ and those at the peaks are around 0.005 (Fig. S6†). This observation is comparable to the reported values for CPL complexes. ${ }^{19}$

\section{Conclusions}

In conclusion, two Schiff base ancillary ligands with $R / S$ chiral carbon atoms were synthesized and two iridium complexes with racemic configuration $(\Lambda / \Delta)-(\mathrm{dfppy})_{2} \operatorname{Ir}($ chty- $R)$ and $(\Lambda / \Delta)$ (dfppy) $)_{2} \operatorname{Ir}($ chty- $S$ ) were obtained. Furthermore, four isomers $\Lambda$-(dfppy $)_{2} \operatorname{Ir}($ chty- $R), \quad \Delta$-(dfppy $)_{2} \operatorname{Ir}($ chty- $R), \quad \Lambda$-(dfppy $)_{2} \operatorname{Ir}($ chty- $S)$ and $\Delta$-(dfppy $)_{2} \operatorname{Ir}($ chty- $S)$ were separated by chiral synthetic HPLC with high ee values. All the isomers were characterized by X-ray single crystal diffraction. In $\Lambda$-(dfppy $)_{2} \operatorname{Ir}($ chty- $R)$ and $\Delta$-(dfppy $)_{2} \operatorname{Ir}($ chty- $S)$, the $\pi-\pi$ stacking is found between the main ligand and the ancillary ligand, but this is not observed in $\Delta$-(dfppy $)_{2} \operatorname{Ir}($ chty- $R)$ and $\Lambda$-(dfppy $)_{2} \operatorname{Ir}($ chty- $S)$ due to disadvantageous steric arrangements of the phenyl ring for packing on chiral carbon atoms. All the isomers have exactly the same steady state photophysical properties, but the ECD spectra are significantly disturbed by the chiral carbon atoms, which give strong evidence for the dual stereogenic centers. The isomers with $\Lambda$ and $\Delta$ configurations show negative and positive CPL signals, respectively, and the $g_{\text {Lum }}$ values are constantly in the scope of $10^{-3}$.

\section{Acknowledgements}

This work was supported by the National Natural Science Foundation of China (21301095, 21371093, 91433113), the Major State Basic Research Development Program 
(2013CB922101), and the Natural Science Foundation of Jiangsu Province (BY2016075-02).

\section{Notes and references}

1 (a) S. Lamansky, P. Djurovich, D. Murphy, F. Abdel-Razzaq, H. E. Lee, C. Adachi, P. E. Burrows, S. R. Forrest and M. E. Thompson, J. Am. Chem. Soc., 2001, 123, 4304; (b) S. Okada, K. Okinaka, H. Iwawaki, M. Furugori, M. Hashimoto, T. Mukaide, J. Kamatani, S. Igawa, A. Tsuboyama, T. Takiguchi and K. Ueno, Dalton Trans., 2005, 1583; (c) C. J. Chang, C. H. Yang, K. Chen, Y. Chi, C. F. Shu, M. L. Ho, Y. S. Yeh and P. T. Chou, Dalton Trans., 2007, 1881; (d) G. J. Zhou, C. L. Ho, W. Y. Wong, Q. Wang, D. G. Ma, L. X. Wang, Z. Y. Lin, T. B. Marder and A. Beeby, Adv. Funct. Mater., 2008, 18, 499; (e) T. Y. Li, X. Liang, L. Zhou, C. Wu, S. Zhang, X. Liu, G. Z. Lu, L. S. Xue, Y. X. Zheng and J. L. Zuo, Inorg. Chem., 2015, 54, 161.

2 Y. C. Zhu, L. Zhou, H. Y. Li, Q. L. Xu, M. Y. Teng, Y. X. Zheng, J. L. Zuo, H. J. Zhang and X. Z. You, Adv. Mater., 2011, 23, 4041.

3 (a) M. Licini and J. A. G. Williams, Chem. Commun., 1999, 1943; (b) L. Huynh, Z. U. Wang, J. Yang, V. Stoeva, A. Lough, I. Manners and M. A. Winnik, Chem. Mater., 2005, 17, 4765; (c) Q. Zhao, T. Y. Cao, F. Y. Li, X. H. Li, H. Jing, T. Yi and C. H. Huang, Organometallics, 2007, 26, 2077.

4 (a) M. X. Yu, Q. Zhao, L. X. Shi, F. Y. Li, Z. G. Zhou, H. Yang, T. Yia and C. H. Huang, Chem. Commun., 2008, 2115; (b) J. L. Liu, Y. Liu, Q. Liu, C. Y. Li, L. N. Sun and F. Y. Li, J. Am. Chem. Soc., 2011, 133, 15276; (c) Y. M. Yang, Q. Zhao, W. Feng and F. Y. Li, Chem. Rev., 2013, 113, 192.

5 P. Hayoz, A. Vonzelewsky and H. Stoecklievans, J. Am. Chem. Soc., 1993, 115, 5111.

6 (a) C. Schaffner-Hamann, A. von Zelewsky, A. Barbieri, F. Barigelletti, G. Muller, J. P. Riehl and A. Neels, J. Am. Chem. Soc., 2004, 126, 9339; (b) F. J. Coughlin, M. S. Westrol, K. D. Oyler, N. Byrne, C. Kraml, E. ZysmanColman, M. S. Lowry and S. Bernhard, Inorg. Chem., 2008, 47, 2039; (c) L. R. Yang, A. von Zelewsky, H. P. Nguyen, G. Muller, G. Labat and H. Stoeckli-Evans, Inorg. Chim. Acta, 2009, 362, 3853; (d) A. Auffrant, A. Barbieri,
F. Barigelletti, J. Lacour, P. Mobian, J. P. Collin, J. P. Sauvage and B. Ventura, Inorg. Chem., 2007, 46, 6911.

7 T. Y. Li, Y. M. Jing, X. Liu, Y. Zhao, L. Shi, Z. Y. Tang, Y. X. Zheng and J. L. Zuo, Sci. Rep., 2015, 5, 14912.

8 (a) M. Suarez and G. B. Schuster, J. Am. Chem. Soc., 1995, 117, 6732; (b) N. P. M. Huck, W. F. Jager and B. L. Feringa, Science., 1996, 273, 1686; (c) C. S. Wang, H. S. Fei, Y. Qiu, Y. Q. Yang, Z. Q. Wei, Y. Q. Tian, Y. M. Chen and Y. Y. Zhao, Appl. Phys. Lett., 1999, 74, 19.

9 M. Schadt, Annu. Rev. Mater. Sci., 1997, 27, 305.

10 (a) R. Farshchi, M. Ramsteiner, J. Herfort, A. Tahraoui and H. T. Grahn, Appl. Phys. Lett., 2011, 98, 162508; (b) I. Žutić, J. Fabian and S. Das Sarma, Rev. Mod. Phys., 2004, 76, 323.

11 (a) X. B. Yin, Z. L. Ye, J. Rho, Y. Wang and X. Zhang, Science, 2013, 339, 1405; (b) C. Wagenknecht, C.-M. Li, A. Reingruber, X.-H. Bao, A. Goebel, Y.-A. Chen, Q. Zhang, K. Chen and J.-W. Pan, Nat. Photonics, 2010, 4, 549.

12 J. F. Sherson, H. Krauter, R. K. Olsson, B. Julsgaard, K. Hammerer, I. Cirac and E. S. Polzik, Nature, 2006, 443, 557.

13 (a) T. Oka and H. Aoki, Phys. Rev. B: Condens. Matter, 2009, 79, 081406(R); (b) Y. H. Wang, H. Steinberg, P. JarilloHerrero and N. Gedik, Science, 2013, 342, 453.

14 G. Mazzeo, M. Fuse, G. Longhi, I. Rimoldi, E. Cesarotti, A. Crispini and S. Abbate, Dalton Trans., 2016, 45, 992.

15 A. C. Chamayou, G. Makhloufi, L. A. Nafie, C. Janiak and S. Ludeke, Inorg. Chem., 2015, 54, 2193.

16 (a) H. Y. Li, T. Y. Li, M. Y. Teng, Q. L. Xu, S. Zhang, Y. M. Jin, X. Liu, Y. X. Zheng and J. L. Zuo, J. Mater. Chem. C, 2014, 2, 1116; (b) Q. L. Xu, C. C. Wang, T. Y. Li, M. Y. Teng, S. Zhang, Y. M. Jing, X. Yang, W. N. Li, C. Lin, Y. X. Zheng, J. L. Zuo and X. Z. You, Inorg. Chem., 2013, 52, 4916.

17 (a) X. J. Liu, B. Yao, Z. L. Zhang, X. F. Zhao, B. H. Zhang, W. Y. Wong, Y. X. Cheng and Z. Y. Xie, J. Mater. Chem. C, 2016, 4, 5787; (b) J. Lin, N. Y. Chau, J. L. Liao, W. Y. Wong, C. Y. Lu, Z. T. Sie, C. H. Chang, M. A. Fox, P. J. Low, G. H. Lee and Y. Chi, Organometallics, 2016, 35, 1813.

18 (a) W. J. Finkenzeller and H. Yersin, Chem. Phys. Lett., 2003, 377, 299; (b) A. F. Rausch, M. E. Thompson and H. Yersin, Inorg. Chem., 2009, 48, 1928.

19 M. Ashizawa, L. Yang, K. Kobayashi, H. Sato, A. Yamagishi, F. Okuda, T. Harada, R. Kuroda and M. Haga, Dalton Trans., 2009, 1700. 